\title{
Ruptured anterior paraclinoid aneurysms
}

\author{
Tetsuyoshi Horiuchi, M.D., Yoshikazu Kusano, M.D., Takehiro Yako M.D., \\ Takahiro Murata, M.D., Yukinari Kakizawa, M.D., and Kazuhiro Hongo, M.D. \\ Department of Neurosurgery, \\ Shinshu University School of Medicine, \\ Matsumoto, Japan
}

Running title: Ruptured (anterior ) “dorsal” paraclinoid aneurysm

Key words: paraclinoid aneurysm, internal carotid artery, surgery

Correspondence to:

Tetsuyoshi Horiuchi, M.D.

Department of Neurosurgery,

Shinshu University School of Medicine,

3-1-1 Asahi, Matsumoto 390-8621, Japan

Telephone: +81-263-37-2690

Fax: +81-263-37-0480

E-mail: tetuyosi@shinshu-u.ac.jp 


\begin{abstract}
The purpose of this study was to evaluate cases of subarachnoid hemorrhage (SAH) from ruptured anterior (“dorsal”) paraclinoid aneurysms. Anterior paraclinoid aneurysms are defined as aneurysms arising from the anterolateral wall of the proximal internal carotid artery without any relationship to an arterial branch. Between 1991 and 2008, a total of 159 patients with 169 paraclinoid aneurysms were treated at the Shinshu University Hospital and its affiliated hospitals. Retrospective analysis was carried out using charts, operation record, operation video, and neuroimagings. 26 patients had anterior paraclinoid aneurysms. Six patients presented with SAH. Three aneurysms were saccular and the others were blister-like aneurysms based on operative findings. Neck laceration or premature rupture frequently happened during the clipping surgery even though the aneurysm was saccular type. The treatment of a ruptured anterior paraclinoid aneurysm is quite difficult. Trapping and bypass would be recommended for such fragile aneurysms.
\end{abstract}




\section{Introduction}

Aneurysms arising from the anterior wall of the internal carotid artery (ICA), so-called “dorsal” wall aneurysm, were originally reported from our institution in 1986 [9]. These aneurysms originate from non-branching sites of the ICA. They often presented with a "blister-like or blood blister-like" configuration. Blister-like aneurysms are characterized by a small hemispherical bulge on angiography and extremely fragile walls that are easily ruptured [1, 8, 9, 10, 13, 14]. Clip surgery alone may provoke aneurysm avulsion and ICA laceration $[4,13]$. Such fragile aneurysms might be treated with trapping $[4,13]$. By contrast, some of anterior (“dorsal”) wall aneurysms presented a saccular configuration with neck that may be clipped [10].

Anterior wall ICA aneurysms usually arose from the distal ICA rather than the paraclinoid region [6, 13]. In 1991, Aldrich [2] firstly reported a case of ruptured anterior (dorsal) paraclinoid aneurysm. The aneurysm was a saccular type with thin wall. Kinouchi and colleagues [6] also presented 7 saccular anterior paraclinoid aneurysms in 2002. These findings are consistent with other studies [5, 11]. However, ruptured anterior paraclinoid aneurysm would be different from unruptured aneurysm based on the author's experiences. In this report, we focused ruptured anterior (“dorsal”) paraclinoid aneurysms and conducted a retrospective analysis of surgically treated anterior paraclinoid aneurysms causing subarachnoid hemorrhage (SAH).

\section{Methods}

This study was performed with the approval of the Shinshu University Institutional Review Board. At the Shinshu University Hospital and its affiliated hospitals, 169 paraclinoid aneurysms (159 patients) were treated between 1991 and 2008. Paraclinoid aneurysms treated with the endovascular treatment were not included in this study. Medical records, radiologic studies, and operation record including operation video were reviewed retrospectively. We defined the dorsal paraclinoid aneurysm as an aneurysm originating from non-branching site and arising from the antero-lateral surface of the intradural ICA proximal to the origin of the posterior communicating artery. According to operative findings, the anterior paraclinoid aneurysms were divided into blister-like and saccular aneurysms. The blister-like aneurysm had dissecting nature without definitive aneurysm neck. 
For statistical analyses, Fisher's exact test or unpaired t-test was used and significant differences were considered at $\mathrm{P}<0.05$.

\section{Results}

Twenty six dorsal paraclinoid aneurysms were found (Table1). Nineteen aneurysms were asymptomatic. All asymptomatic aneurysms (incidental or associated aneurysms) were saccular aneurysms according to operation record and/or operation video and they were obliterated using clips. Six aneurysmal ruptures caused an SAH (Table 1 and 2) and one aneurysm induced a visual disturbance. Type of aneurysm was different between asymptomatic and ruptured aneurysms (Table 1).

Table 2 summarized clinical characteristics of ruptured dorsal paraclinoid aneurysms. The age of patients ranged from 25 to 74 (average 51) years with female preponderance. Three aneurysms were not detected out on initial angiograms. Aneurysms developed in both sides and the size varied from 3 to 9 (average 4.8) $\mathrm{mm}$. Three were blister-like aneurysms and the other three aneurysms were saccular type. Usually, preoperative judgment of saccular or blister-like aneurysm was difficult on preoperative angiograms.

Basically, ruptured dorsal ICA aneurysms had been treated with clipping or wrap-clipping before 2007. Currently, trapping with a high-flow bypass is the first choice treatment for the ruptured dorsal ICA aneurysm because of high rate of premature rupture during clipping surgery [4]. Previous cases (1 to 5) underwent clipping or wrap-clipping until 2006. In four cases of 5 (80\%), neck laceration or premature rupture happened during clipping surgery. Recent Case 6 was treated with trapping and double insurance bypass [3].

\section{Illustrative cases}

Case 2

This 66-yaer-old woman presented with a massive SAH caused by a "dorsal” aneurysm at the left ICA (Figure 1). A left fronto-temporal craniotomy was made. Since the aneurysm would be embedded into the frontal lobe, the anterior temporal approach was used not to retract the frontal lobe. The aneurysmal neck was exposed after opening the medial sylvian fissure. The aneurysm seemed to be saccular type (Figure 2). A bayonet clip was successfully applied for obliteration. Severe vasospasm developed postoperatively and she died. 


\section{Case 3}

This 57-year-old woman suffered an SAH. The aneurysm was not found on the initial angiogram and became visible on follow-up angiograms (Figure 3). The patient underwent the clipping surgery on Day 25. A left frontotemporal craniotomy was performed with preparation for the neck carotid arteries for possible occlusion. The sylvain fissure was carefully opened so as not to retract the frontal lobe. The anterior clinoid process was partially removed to secure proximal clipping of the parent artery. The proximal and distal neck was dissected under temporary occlusion of the parent artery. The aneurysm seemed to be saccular one. During clip placement, the neck tear in the distal part happened. The aneurysmal neck including the tear was successfully eliminated with two straight clips. Two straight clips were applied in a parallel fashion to narrow the lumen of the parent artery (Figure 3).

Case 6

This 29-year-old woman was admitted under diagnosis of an SAH. Subsequent three-dimensional computed tomographic angiography disclosed no aneurysms. A follow-up workup demonstrated an irregular shaped dorsal paraclinoid aneurysm at the right ICA (Figure 4). A right frontotemporal craniotomy was done with preparation of the neck carotid arteries for anastomosis and proximal control. To avoid the ischemic complication during radial artery bypass, the superficial temporal artery (STA) was firstly anastomosed to a cortical branch (M4) of the middle cerebral artery (MCA) [3]. A radial artery-external carotid artery-M2 portion of MCA bypass was performed. Then, the sylvian fissure was carefully opened with minimum retraction of the frontal lobe. The fragile neck was visualized and the aneurysmal dome was embedded into the frontal lobe (Figure 4). The aneurysm was successfully trapped with preservations of the posterior communicating and ophthalmic arteries (Figure 5 and 6).

\section{Discussion}

\section{Anterior (“Dorsal”) ICA aneurysm:}

These branchless aneurysms have been also called superior wall aneurysm or ICA trunk aneurysms $[7,9,10]$ and include the blister type and the saccular type [10]. In some cases, it is difficult to distinguish the saccular type from the blister type aneurysms based on preoperative angiograms $[9,14]$. Fragile wall is also reported in the dorsal aneurysm with 
saccular configuration [7, 9, 10]. Therefore, in both types of anterior ICA aneurysm, premature rupture easily happens resulting in poor outcome [4, 9]. Although some saccular type aneurysms would be easily clipped like ordinary berry aneurysm [10], vascular neurosurgeon should keep in mind that the importance of recognizing anteior ICA aneurysm refers to the difficulty of clipping $[9,14]$. Wrapping or clipping on wrapping with material have risks of rerupture and regrowth of aneurysms.

\section{Ruptured anterior (“dorsal”) paraclinoid aneurysm:}

Anterior parclinoid aneurysms are extremely rare. Kinouchi et al. [6] reported 7 cases harboring anterior paraclinoid aneurysms. Their series consisted of 6 asymptomatic aneurysms and ruptured one. All aneurysms were saccular type and they were well clipped through the pterinoal route. Their results were consistent with the present asymptomatic aneurysms. However, type of ruptured anterior paraclinoid aneurysms was different from asymptomatic aneurysm in the present study. Premature rupture or neck laceration frequently happened during clipping surgery. Three of 6 patients had blister-like aneurysms and simple clippings were not achieved in all cases. Therefore, ruptured anterior paraclinoid aneurysms seemed to be the same category of the dorsal ICA aneurysm originating from the distal ICA.

\section{Treatment of ruptured anterior paraclinoid aneurysm:}

Various surgical tactics and strategies of “dorsal” ICA aneurysms have been reported including clipping with parent artery stenosis, wrapping, clip placement over the wrapping material, trapping, and endovascular obliteration [1, 7, 8, 10,12]. All surgical procedures pose a high risk of intraoperative rupture including ICA laceration and postoperative regrowth and rebleeding. Park et al. [12] reported results of endovascular treatments of blister-like aneurysm. Coil embolizations resulted in the aneurysm regrowth in all 4 cases. Trapping would be the safest procedure for a “dorsal” ICA aneurysm. However, the trapping decreases or abolishes anterograde blood flow of the ICA. Meling and colleagues [8] reported that ICA trap made the patients with ruptured blister-like aneurysm poor outcome even though there was adequate collateral blood flow. They also mentioned that ICA trap could not be safely done when the cerebral vasospasm occurred after surgery. Therefore, cerebral revascularization should be necessary even though the collateral blood flow might be sufficient during trapping procedure. High-flow bypass is recommended rather than 
STA-MCA bypass to restore the blood flow supplied by the ICA [8]. In addition, trapping of ICA has a risk to develop a de novo aneurysm at the contralateral side.

Currently we select trapping with double bypass (high-flow bypass following low-flow bypass) [3] for the treatment of ruptured dorsal ICA aneurysm including at the paraclinoid region. At this moment, trapping with bypass might be the best treatment option of fragile aneurysms with minimum complications [4].

\section{Conclusions}

Ruptured anterior (“dorsal”) paraclinoid aneurysms are rare and quite different from unruptured anterior paraclinoid aneurysms. Based on our results, we recommend surgical trapping with high-flow bypass for the treatment of ruptured anterior paraclinoid aneurysms including a saccular type aneurysm because of high rate of premature rupture or neck laceration. 


\section{Figure legends}

Figure 1: Computed tomography (A and B) demonstrating a subarachnoid hemorrhage with intracerebral hematoma. Preoperative anteroposterior (C) and oblique (D) projections of the left carotid artery injection revealing a “dorsal” paraclinoid aneurysm.

Figure 2: Intraoperative photographs (A: before clipping, B: after clipping) showing the operative site. Note that the aneurysm $(*)$ seemed to be saccular type.

Figure 3: Anteroposterior projections of serial left carotid angiograms (A: initial angiogram, B: preoperative angiogram, C: postoperative angiogram). Initial angiogram did not detect a “dorsal” paraclinoid aneurysm discovered on a follow-up angiogram. The aneurysm was successfully clipped with parent artery stenosis.

Figure 4: The right carotid artery injection (A: anteroposterior view, B: 3-dimensional image) showing an irregular shaped “dorsal” paraclinoid aneurysm (arrowhead). Intraoperative photographs (C and D) through the pterional approach showing a blister-like aneurysm (*) which was trapped with clips. ICA: internal carotid artery, II: optic nerve, PCoA: posterior communicating artery.

Figure 5: The postoperative right carotid artery injection (A: anteroposterior view, B and C: lateral view) revealing the trapping of the aneurysm with preservation of the ophthalmic artery (Oph. A) and patency of the anastomoses between the superficial temporal artery (STA) and the middle cerebral artery (MCA) and between the radial artery (RA) and the MCA.

Figure 6: The postoperative right vertebral injection (A: oblique view, B: lateral view) showing the anterior cerebral artery fed by the posterior communicating artery. 


\section{Reference List}

1. Abe M, Tabuchi K, Yokoyama H, Uchino A (1998) Blood blisterlike aneurysms of the internal carotid artery. J Neurosurg 89:419-424

2. Aldrich F (1991) Anterior (dorsal) paraclinoid aneurysm: case report. Surg Neurol 35:374-376

3. Hongo K, Horiuchi T, Nitta J, Tanaka Y, Tada T, Kobayashi S (2003) Double-insurance bypass for internal carotid artery aneurysm surgery. Neurosurgery 52:597-602

4. Hongo K, Satoh A, Kakizawa Y, Miyahara T, Tanaka Y, Sugiyama T (2006) The nationwide surveillance on the dorsal aneurysm of the internal carotid artery. Part 1: analysis of the factors affecting the poor outcome. Surg Cereb Stroke (Jpn) 34:366-371

5. Horiuchi T, Tanaka Y, Kusano Y, Yako T, Sasaki T, Hongo K (2009) Relationship between the ophthalmic artery and the dural ring of the internal carotid artery. Clinical article. J Neurosurg 111:119-123

6. Kinouchi H, Mizoi K, Nagamine Y, Yanagida N, Mikawa S, Suzuki A, Sasajima T, Yoshimoto T (2002) Anterior paraclinoid aneurysms. J Neurosurg 96:1000-1005

7. Kobayashi S, Hongo K, Shigeta H, Goel A (1997) Dorsal internal carotid artery aneurysms, in Kobayashi S, Goel A, Hongo K (eds): Neurosurgery of complex tumors and vascular lesions. London, Churchill Livingstone, pp 37-46.

8. Meling TR, Sorteberg A, Bakke SJ, Slettebo H, Hernesniemi J, Sorteberg W (2008) Blood blister-like aneurysms of the internal carotid artery trunk causing subarachnoid hemorrhage: treatment and outcome. J Neurosurg 108:662-671

9. Nakagawa F, Kobayashi S, Takemae T, Sugita K (1986) Aneurysms protruding from the 
dorsal wall of the internal carotid artery. J Neurosurg 65:303-308

10. Ogawa A, Suzuki M, Ogasawara K (2000) Aneurysms at nonbranching sites in the surpaclinoid portion of the internal carotid artery: internal carotid artery trunk aneurysms. Neurosurgery 47:578-583

11. Onoda K, Tokunaga K, Sugiu K, Ono S, Date I (2006) [Direct surgery for paraclinoid aneurysm arising from the anterolateral wall of the internal carotid artery]. No Shinkei Geka 34:267-272

12. Park JH, Park IS, Han DH, Kim SH, Oh CW, Kim JE, Kim HJ, Han MH, Kwon OK (2007) Endovascular treatment of blood blister-like aneurysms of the internal carotid artery J Neurosurg 106:812-819

13. Satoh A, Hongo K, Sugiyama T, Ishihara S, Yamane F, Kakizawa Y (2006) The nationwide surveillance on the dorsal aneurysms of the internal carotid artery. Part 2: Study on the surgical treatment in hemorrhagic cases. Surg Cereb Stroke (Jpn) 34:372-376

14. Shigeta H, Kyoshima K, Nakagawa F, Kobayashi S (1992) Dorsal internal carotid artery aneurysms with special reference to angiographic presentation and surgical management. Acta Neurochir (Wien ) 119:42-48 
Table 1. Comparison of asymptomatic and ruptured anterior paraclinoid aneurysms.

$$
\text { Asymptomatic an. Ruptured an. }
$$

Characteristics

$$
\text { P value }
$$

$$
(\mathrm{n}=19) \quad(\mathrm{n}=6)
$$

\begin{tabular}{lccc}
\hline mean age (yrs) & $56.8 \pm 12.4$ & $51.0 \pm 19.8$ & NS* \\
sex (M/F) & $3: 16$ & $1: 5$ & NS† \\
type of aneurysm & $19: 0$ & $3: 3$ & $0.009 \dagger$ \\
(saccular /blister-like) & $9: 10$ & $3: 3$ & NS $†$ \\
side of aneurysm (R/L) & $4.9 \pm 1.6$ & $4.8 \pm 2.2$ & NS* \\
mean aneurysm size (mm) & & & \\
\hline
\end{tabular}

Data are presented as the means \pm standard deviation.

an: aneurysm, n: number of patient, R: right, L: left, M: male, F: female, *: Unpaired t-test, $\uparrow$ : Fisher's exact test, NS: not significant. 
Table 2: Characteristics of 6 patients with ruptured anterior paraclinoid aneurysms

\begin{tabular}{|c|c|c|c|c|c|c|c|c|}
\hline $\begin{array}{l}\text { Case } \\
\text { No. }\end{array}$ & $\begin{array}{l}\text { Age(yrs) } \\
\text { /Sex }\end{array}$ & $\begin{array}{l}\text { WFNS } \\
\text { Grade }\end{array}$ & Side & $\begin{array}{l}\text { Size } \\
(\mathrm{mm})\end{array}$ & Type & $\begin{array}{l}\text { Surgical } \\
\text { treatment }\end{array}$ & $\begin{array}{c}\text { Outcome } \\
\text { (GOS) }\end{array}$ & Other characteristics \\
\hline 1 & $25 / F$ & I & $\mathrm{R}$ & 9 & Saccular & Clipping & GR & $\begin{array}{l}\text { Premature rupture } \\
\text { Not revealed on initial angiograms }\end{array}$ \\
\hline 2 & $66 / F$ & $\mathrm{~V}$ & $\mathrm{~L}$ & 4 & Saccular & Clipping & $\mathrm{D}$ & Severe vasospasm \\
\hline 3 & $57 / F$ & II & $\mathrm{L}$ & 5 & Saccular & Clipping & GR & $\begin{array}{l}\text { Neck laceration } \\
\text { Not revealed on initial angiograms }\end{array}$ \\
\hline 4 & $55 / \mathrm{M}$ & I & $\mathrm{L}$ & 3 & Blister-like & $\begin{array}{l}\text { Wrap } \\
\text {-clipping }\end{array}$ & GR & Premature rupture \\
\hline 5 & $74 / F$ & I & $\mathrm{R}$ & 3 & Blister-like & $\begin{array}{l}\text { Wrap } \\
\text {-clipping }\end{array}$ & SD & Neck laceration \\
\hline 6 & $29 / F$ & I & $\mathrm{R}$ & 5 & Blister-like & $\begin{array}{c}\text { Trapping and } \\
\text { bypass }\end{array}$ & GR & $\begin{array}{l}\text { Drug allergy } \\
\text { Not revealed on initial angiograms }\end{array}$ \\
\hline
\end{tabular}

WFNS: World Federation of Neurosurgical Societies, M: male, F: female, R: right, L: left, GOS: Glasgow outcome scale, GR: good recovery,

SD: severe disability, D: dead. 

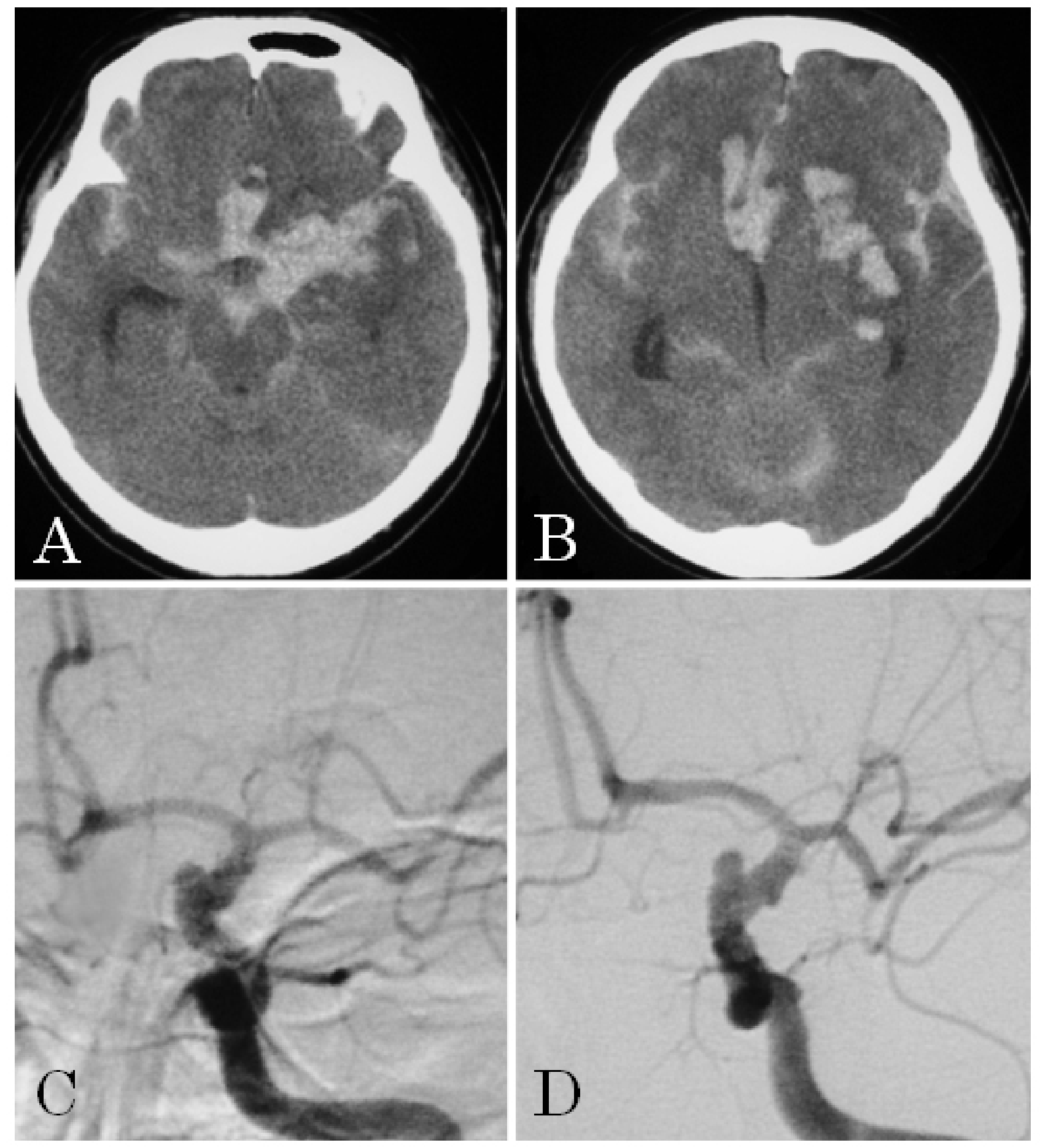

Figure 1 

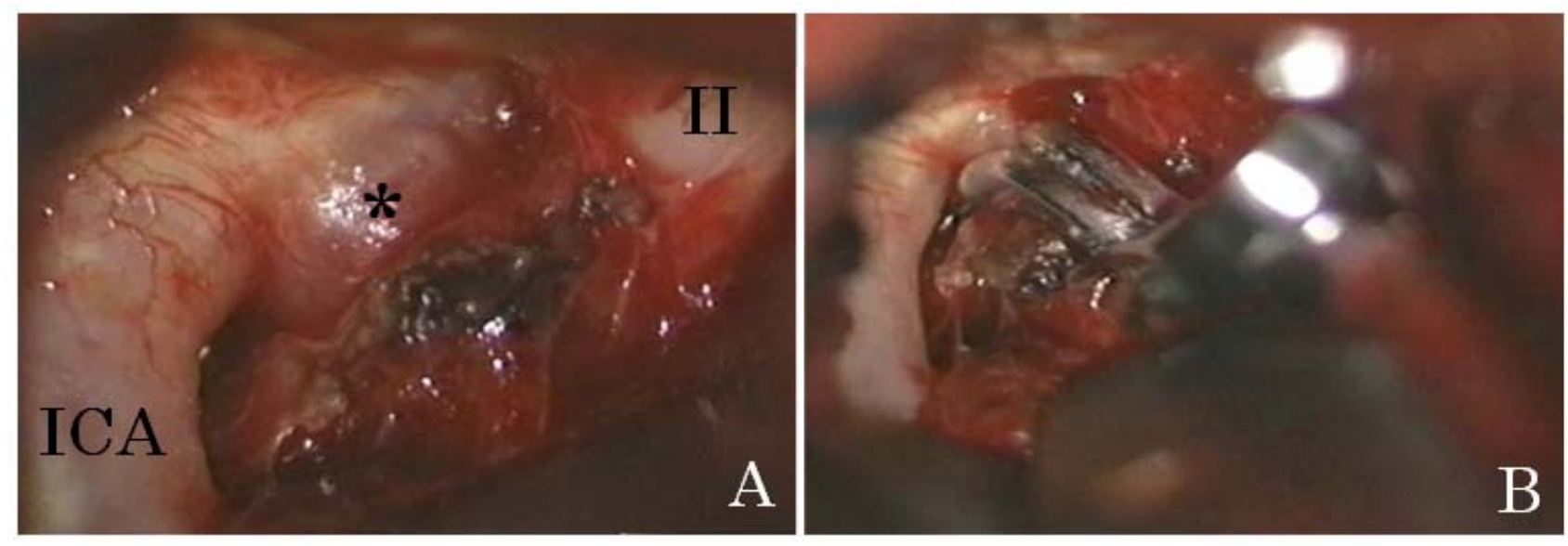

Figure 2
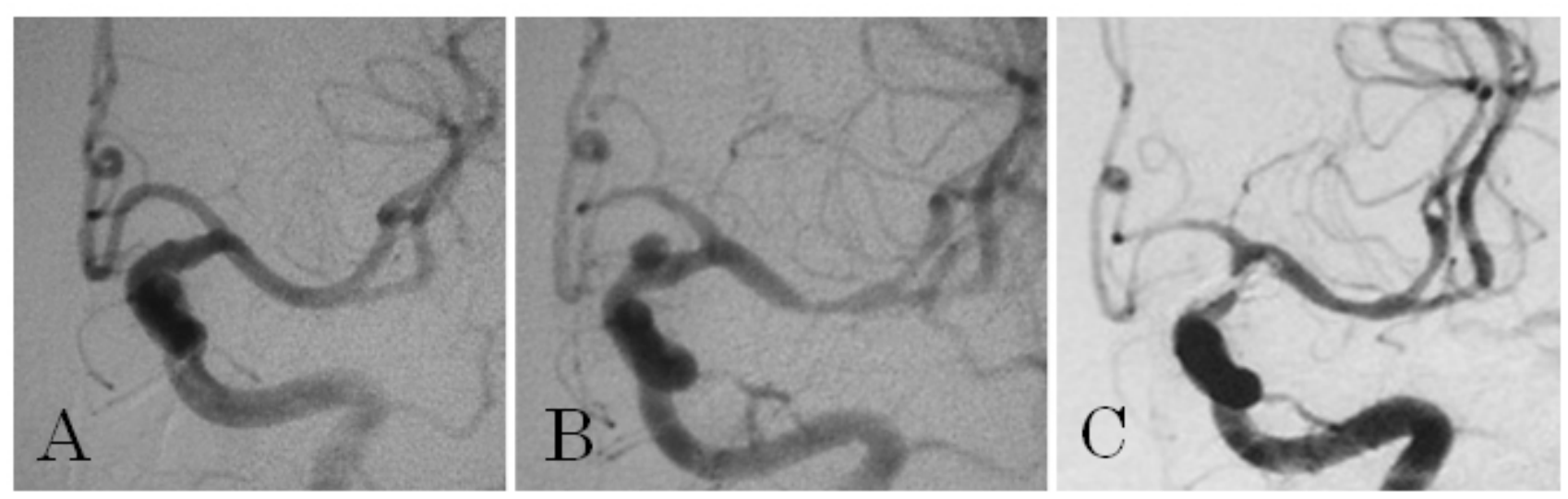

Figure 3 


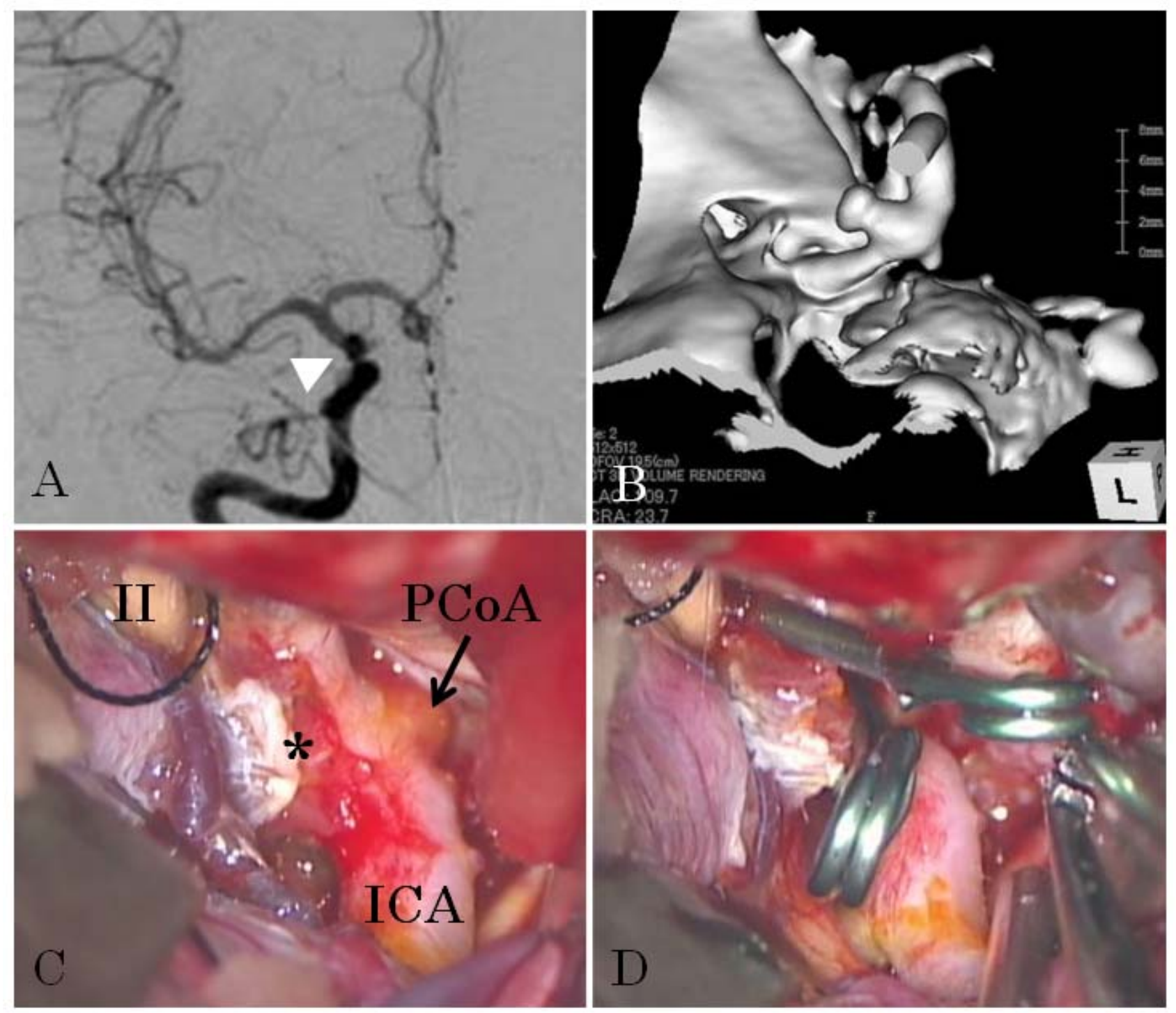

Figure 4
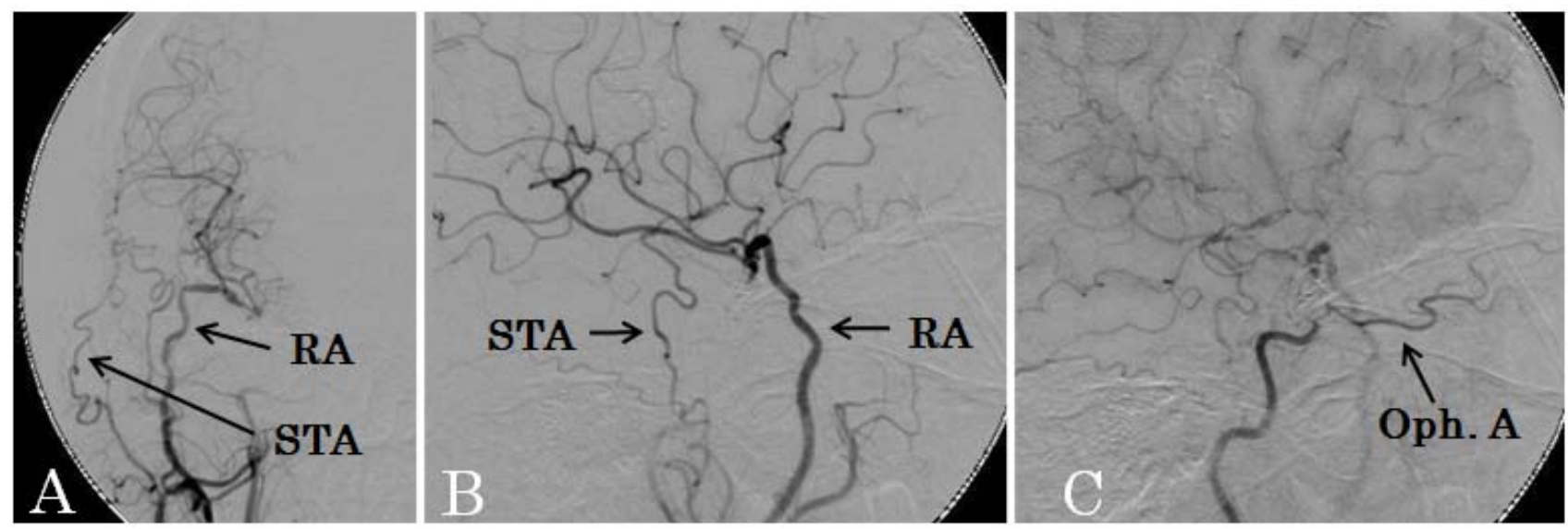

Figure 5 


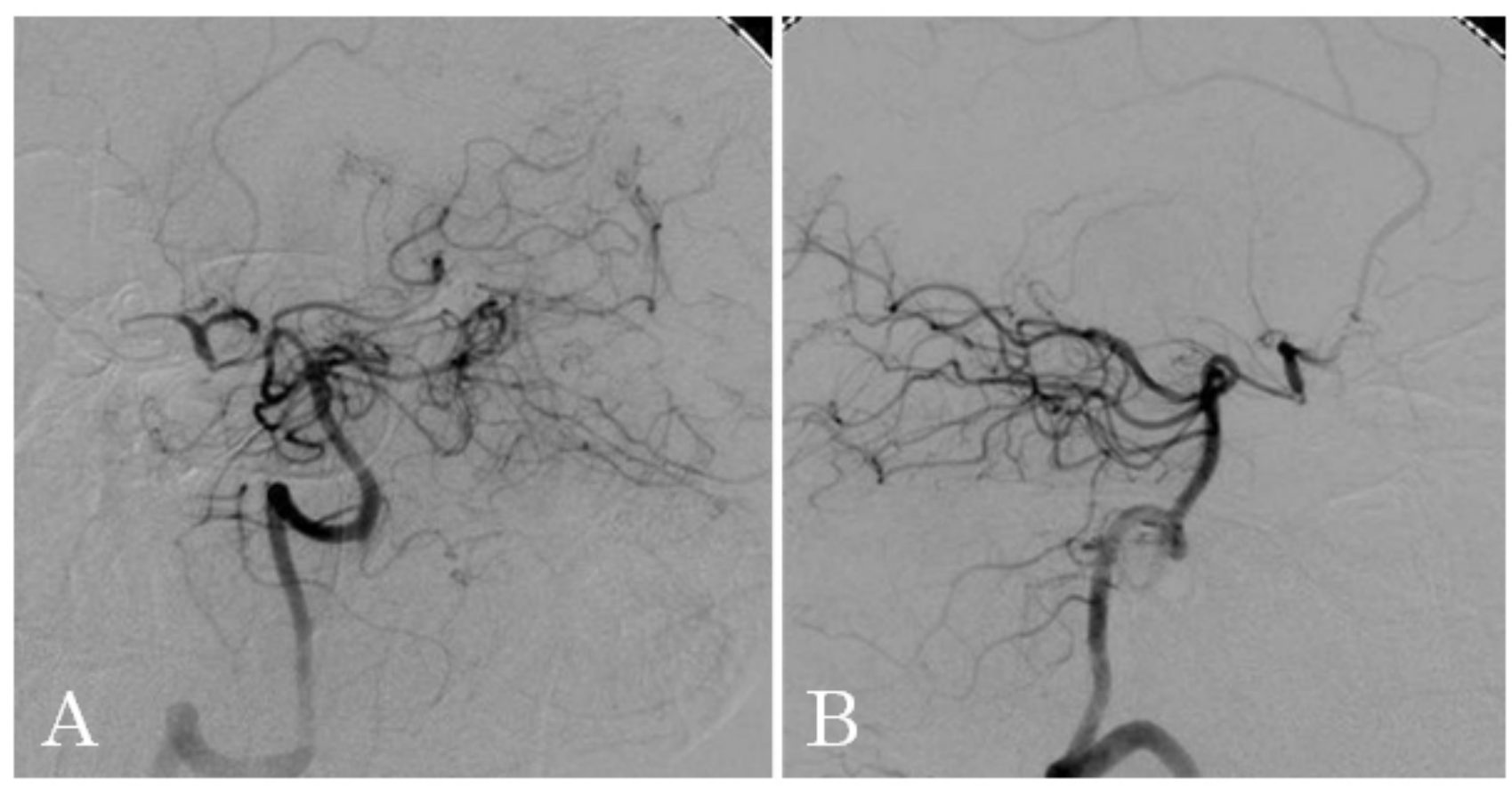

Figure 6 